\title{
Design of Hard Compaction Rollers for Automated Fiber Placement on Complex Mandrel Geometries
}

\author{
Tom T. Zacchia, Farjad Shadmehri, Jeffrey Fortin-Simpson and Suong V. Hoa \\ Concordia Center for Composites (CONCOM), Department of Mechanical, Industrial and Aerospace Engineering, Concordia \\ University, Montreal H3G1M8, Canada
}

\begin{abstract}
This paper presents a methodology for designing hard compaction rollers for an AFP (automated fiber placement) head with a hot gas torch heating system for laying thermoplastic CFRP (carbon fiber reinforced plastic) tapes. Some manufacturing defects and alternative designs are reviewed to highlight the importance of AFP compaction roller conformity and surface adhesion. The formulation of a general parametric description of the design challenge is derived and adequately addresses the problem on a tow-to-tow basis.
\end{abstract}

Key words: AFP, CFRP, compaction roller.

\section{Introduction}

Manufacturers of aircrafts widely use CFRP (carbon fiber reinforced plastic) to produce large parts, such as airfoil covers and fuselages. An example of such is the use of automated fiber placement manufacturing to produce Boeing's 787 Dreamliner's fuselage [1]. AFP (automated fiber placement) is a highly flexible and effective process for manufacturing parts at a high rate of production. However, with parts of increasing geometrical complexity, there are difficulties for a single roller with fixed geometry to provide uniform pressure to compact the tape onto the surface of the part. It is necessary either to have a conformable roller that can adjust its geometry to fit the geometry of the surface of the part, or to have multiple rollers with different geometries that correspond to the geometries at different locations of the part. The objective of this paper is to introduce a methodology for designing hard AFP compaction rollers.

\section{Thermoplastics Manufacturing Defects and Literautre Survey}

The quality of the final product is highly dependent

Corresponding author: Suong Van Hoa, Ph.D, professor, research field: composites. Email: hoasuon@encs.concordia.ca. on a number of manufacturing parameters, such as temperature, pressure and lay-up speed (see Fig. 1). Within the context of this study, pressure and consolidation during the manufacturing process are the primary concerns.

During the manufacturing process pressure is applied after temperature is increased. The sustained pressure during the solidification phase of the process prevents the fiber network from springing back [2]. Under adequate pressure, suppressing fiber spring back minimizes voids that exist between individual layers. Pressure also plays a critical role in the development of intimate contact between plies, which in turn is an important parameter for autohesion. In the most severe case, inadequate conformity between the AFP compaction roller and the mandrel's surface can dramatically reduce pressure applied to the CFRP tape and localize the force (see Fig. 2).

Denkena, Schmidt, and Weber introduced a modular AFP laying head in 2016, which includes a unique set of height adjustable compaction rollers that are pneumatically controlled, thus increasing the flexibility of the design (see Fig. 3) [3]. The compaction rollers used are made of a flexible material, as it is best suited for thermoset CFRP manufacturing, and heating is done with a laser. Qualitatively, experiments conducted 


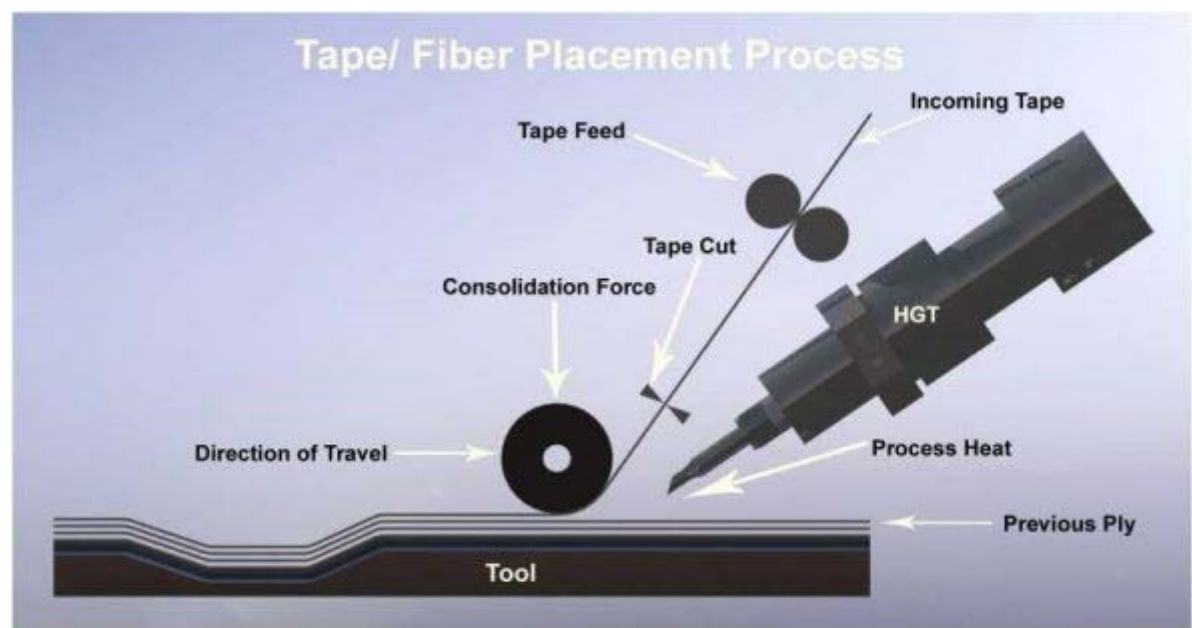

Fig. 1 Fiber placement illustration (photo courtesy of Automated Dynamics).

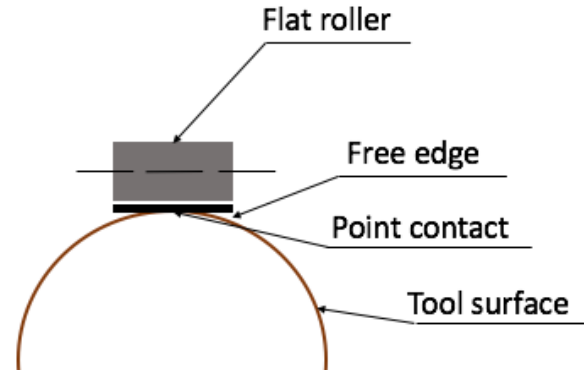

Fig. 2 Illustration of lack of roller and mandrel curvature conformity.

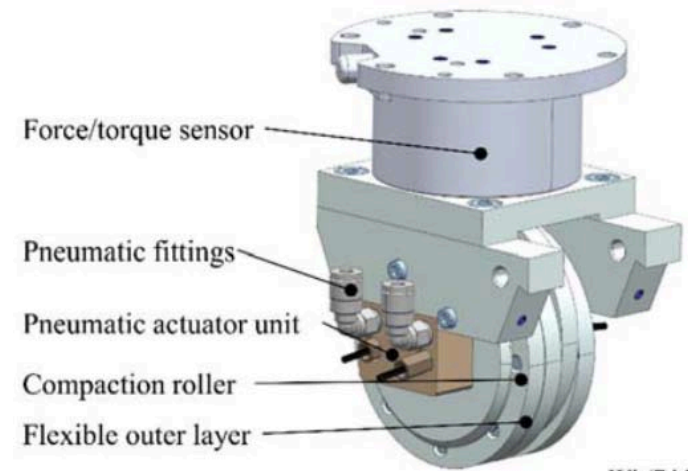

$\mathrm{Wb} / 74419$ ○ IFW

Fig. 3 Modular compaction unit [3].

have validated the effectiveness of the compaction unit both on flat and contoured surfaces. For thermoplastic manufacturing that employs a hot gas torch heating element, hard rollers are better suited to perform the task.

NASA's Phase II SBIR program led to the development of a number of innovative conformable compactors [4]. Research published by Lamontia,
Gruber, Waibel, Cope, and Hulcher included a study on defects caused by poor compactor to surface conformity as well as the design of a highly conformable compaction system. This study highlights that when compactors lack sufficient conformity with the tool surface, the time/temperature/pressure requirements for high-quality laminates are not fulfilled due to the concentration of forces exerted on high points of the tape [4]. An example of one of the high conformity compactor designs can be seen in Fig. 4. This design features 76 heated segments that are covered in shims, which protect the fibers during manufacturing. The compactors were constructed and tested, however the authors did not report the results of those experiments in their study.

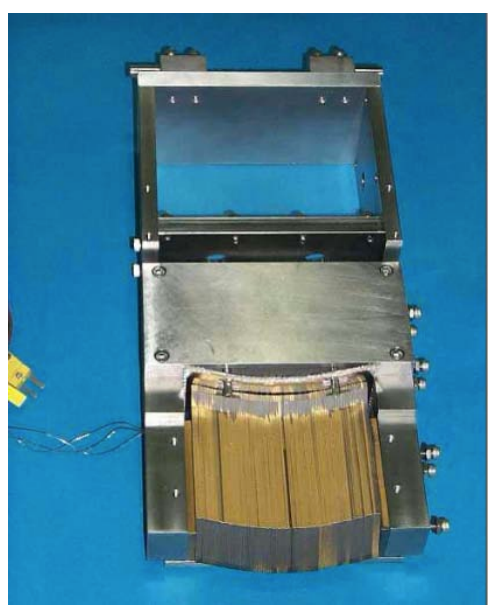

Fig. 4 Hot line compactor featuring 76 heated segments [4]. 
To the best of the authors' knowledge there exists very little research describing an approach to designing hard compaction rollers for thermoplastic CFRP manufacturing; this is the motivation behind the study.

\section{Methodology}

A cylindrical mandrel with a radius of $R s$ and a compaction roller with a profile radius of $R \mathrm{i}$ was used to formulate a general equation to describe the adherence of the two components: with $R \mathrm{i}>R \mathrm{~s}$. A local coordinate system was positioned at the center of the cylinder. However, in Fig. 5 the axes are translated onto the surface of the tape to better describe their directions: $\mathrm{x}$ (normal to the surface), $\mathrm{y}$ (transverse to the layup direction) and $\mathrm{z}$ (in the layup direction).

In the XY plane, the roller's profile and instantaneous curvature at the tool's surface was represented by two concentric circles centered at the origin (see Fig. 6). Compression of the tape during manufacturing can be modeled by translating the circle of smaller curvature, $R \mathrm{~s}$, by $\Delta X$ (Fig. 10). The value of $\Delta X$ is such that the tape is at its minimum thickness under compression, $C$. The aforementioned variable can be determined experimentally.

$$
\Delta X=(R i-R s)-C
$$

With reference to Fig. 7, a number of variables are needed in order to fully describe the distance of the gap that lies between the roller and the mandrel, at the edge of the tape. These are given in Table 1 . The angle $\theta$ (either for the roller or for the surface) is given by,

$$
\theta=\sin ^{-1} \frac{w}{2 R}
$$

The coordinates of the contact point between the roller and the edge of the tape can be described as,

$$
\left[\begin{array}{l}
x_{i} \\
y_{i}
\end{array}\right]=R_{i}\left[\begin{array}{c}
\cos \left(\theta_{i}\right) \\
\frac{w}{2 \cdot R_{i}}
\end{array}\right]
$$

The coordinates of the contact point between the mandrel surface and the edge of the tape are,

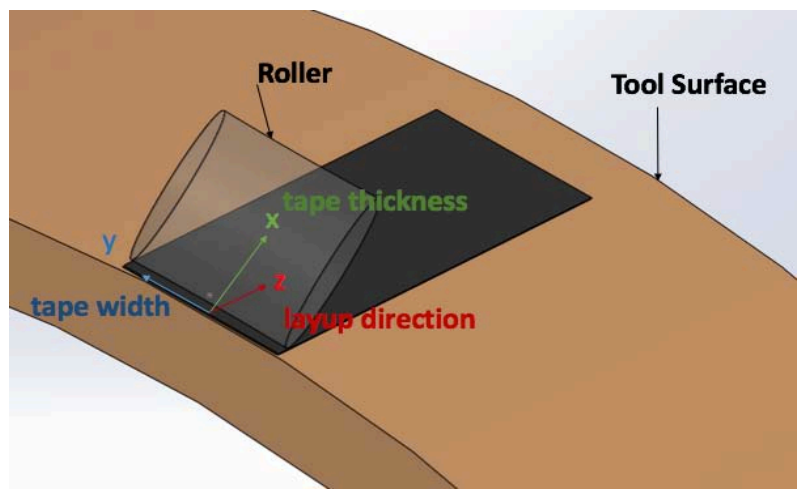

Fig. 5 Coordinate system (shifted).

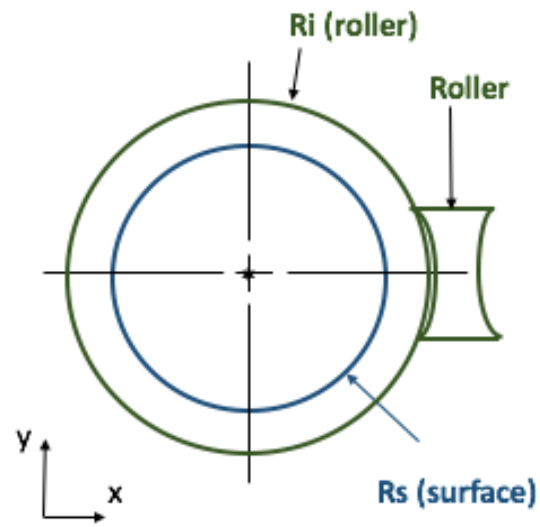

Fig. 6 Concentric circles in design space.

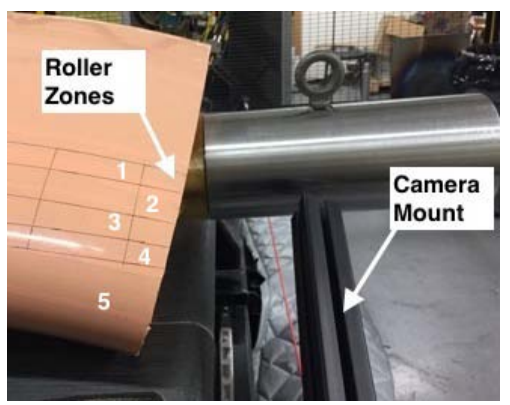

Fig. 7 Boundary conditions of roller curvature.

Table 1 Variables used.

\begin{tabular}{ll}
\hline Variable & Description \\
\hline$C$ & Minimum allowable thickness under compression \\
$w$ & Width of one tow \\
$t$ & $\begin{array}{l}\text { Gap between roller and mandrel surface et edge of } \\
\text { tape }\end{array}$ \\
$h$ & Thickness of one tow/tape \\
$R \mathrm{~s}$ & Radius of curvature of surface \\
$R \mathrm{i}$ & $\begin{array}{l}\text { Radius of curvature of compaction roller } \\
x_{\mathrm{S}}\end{array}$ \\
& $\begin{array}{l}\text { x coordinate corresponding to position on surface } \\
x_{\mathrm{i}}\end{array}$ \\
\hline
\end{tabular}




$$
\left[\begin{array}{l}
x_{s} \\
y_{s}
\end{array}\right]=R_{s}\left[\begin{array}{c}
\cos \left(\theta_{s}\right) \\
\frac{w}{2 \cdot R_{s}}
\end{array}\right]
$$

As previously mentioned $R \mathrm{~s}$ has to be translated by $\Delta X$, the result is a new set of variables ( $\mathrm{x}_{\mathrm{s}}$ ' and $\left.\mathrm{y}_{\mathrm{s}}{ }^{\prime}\right)$.

$$
\left[\begin{array}{l}
x_{s}^{\prime} \\
y_{s}^{\prime}
\end{array}\right]=\left[\begin{array}{l}
x_{s} \\
y_{s}
\end{array}\right]+\left[\begin{array}{c}
\Delta X \\
0
\end{array}\right]
$$

The gap between the roller and the surface of the mandrel at the edge of the tape is,

$$
\begin{gathered}
t=x_{i}-x_{s}{ }^{\prime} \\
t=R_{i} \cos \left(\theta_{i}\right)-\left[R_{s} \cos \left(\theta_{s}\right)+(R i-R s-C)\right] \\
t-C=R_{s}\left(1-\cos \theta_{s}\right)-R_{i}\left(1-\cos \theta_{i}\right)
\end{gathered}
$$

The quantity $(t-C)$ represents the lack of desired compaction. The condition $t / C=1$ represents optimal compaction, while $t / h=1$ represents no compaction. If an allowable minimum compaction (say $t / h=\alpha$ ) is specified, then the profile of the roller can be designed such that $C<t<\alpha h$.

\section{Experimental Investigation}

The set up used for preliminary experimental investigations is seen in Fig. 7. The mandrel's surface is divided into 5 segments, each with a unique range of surface curvature, and 6 compaction rollers were manufactured. An extruded T slot bar was fixed onto a tripod and the assembly was positioned such that the camera's lens is parallel to the subject being captured.

Images were then processed using ImageJ. Fig. 8 depicts an area of 16,362 pixels, which serves as a reference when calculating the percentage of the total area occupied by the gap, if any exits. The aforementioned gap area was calculated by drawing a perimeter encapsulating the gap using ImageJ's polygon tool, see Fig. 9.
The results recorded in Table 2 highlight the successful application of Eq. (6), since the rollers

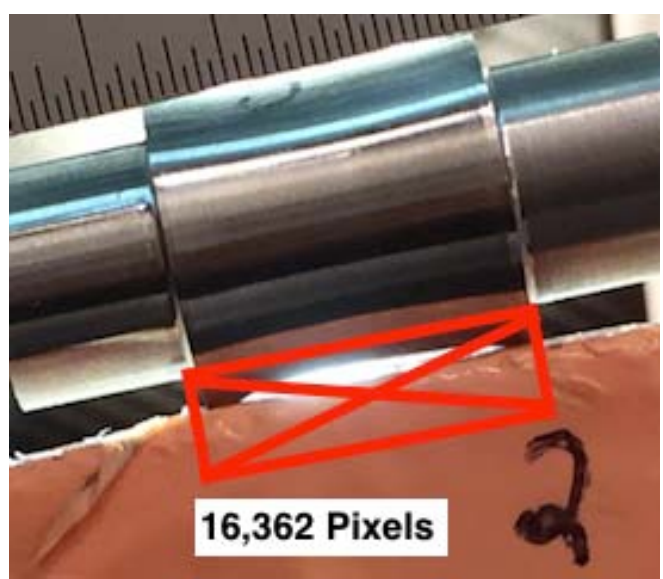

Fig. 8 Reference area.

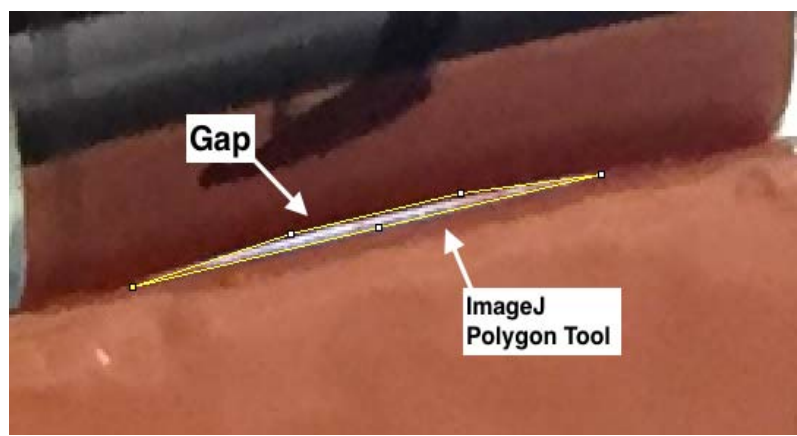

Fig. 9 Calculating gap area (yellow polygon).

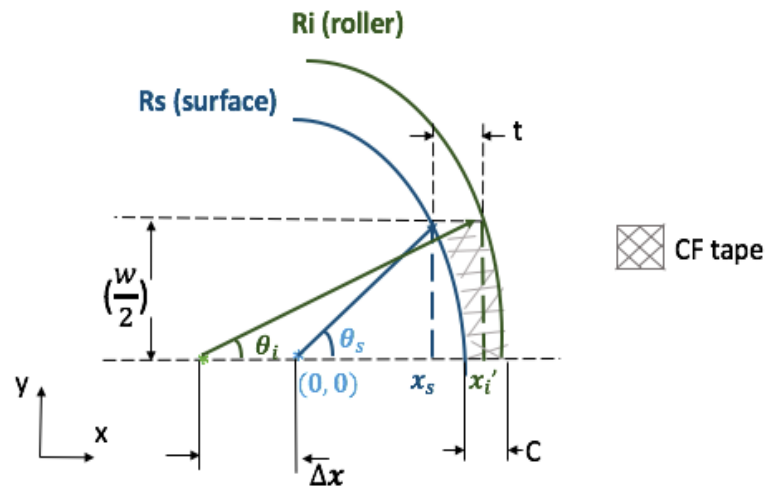

Fig. 10 Boundary conditions of roller curvature.

Table 2 Percentage area occupied by gaps.

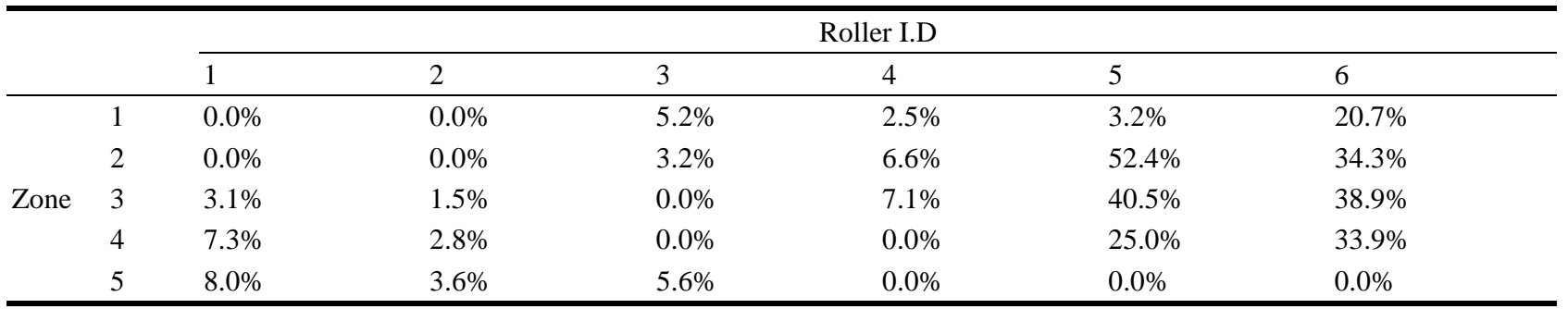


conform well within their intended range of surface curvatures, e.g. roller 1 conforms to segment 1 . In addition, it was observed that even in the least severe case, when the gap area was only $1.5 \%$ of the reference area, the gap distance, $t$, measured in Image $J$ exceeded the thickness of the CFRP tape $(0.127 \mathrm{~mm})$.

\section{Conclusion}

A methodology for calculating an appropriate hard compaction roller profile curvature for a given tool surface curvature, used during thermoplastic AFP processing, has been presented. Eq. (6) can be used to guide the design of compaction rollers for geometries of varying curvatures.

Initial experimental investigations validate Eq. (6) based on the conformity study that was performed in Section 4. Future investigations include using the rollers to manufacture a CFRP component on the same mandrel and study manufacturing defects. The manufacturing process will consist of stopping the AFP head when a particular roller no longer adequately conforms to the tool surface and changing it for a more appropriate compaction roller.

\section{Acknowledgement}

Financial support from the Natural Sciences and Engineering Research Council of Canada is appreciated.

\section{References}

[1] Sloan, J. 2008. "ATL and AFP: Defining the Megatrends in Composite Aerostructures.” High Performance Composites 16 (4): 68.

[2] Hoa, S. 2017. Principles of the Manufacturing of Composite Materials. 2nd ed. DEStech Publications, Inc., pp. 329-80.

[3] Denkena, B., Schmidt, C., and Weber, P. 2016. "Automated Fiber Placement Head for Manufacturing of Innovative Aerospace Stiffening Structure.” Procedia Manufacturing 6: 96-104.

[4] Lamontia, M., Gruber, M., Waibel, B., Cope, R., and Hulcher, B. 2002. "Conformable Compaction System Used in Automated Fiber Placement of Large Composite Aerospace Structures.” www.researchgate.net, January 2002. 